\title{
The effect of melatonin on body mass and behaviour of rats during an exposure to microwave radiation from mobile phone
}

\author{
Sokolovic $\mathrm{D}^{1}$, Djordjevic B ${ }^{1}$, Kocic $\mathrm{G}^{1}$, Babovic $\mathrm{P}^{2}$, Ristic $\mathrm{G}^{3}$, Stanojkovic $\mathrm{Z}^{4}$, Sokolovic DM ${ }^{4}$, \\ Veljkovic $A^{1}$, Jankovic $A^{5}$, Radovanovic $Z^{6}$
}

Department of Biochemistry, Medical Faculty in Nis, University of Nis, Nis, Serbia. soko@medfak.ni.ac.rs

\begin{abstract}
Background: Microwave radiation (MW) produced by wireless telecommunications and a number of electrical devices used in household or in healthcare institutions may cause various disorders in human organism. On the other hand, melatonin is a potent antioxidant, immunostimulator and neuromodulator. The aim of this research was to determine body mass and behaviour changes in rats after a chronic microwave exposure, as well as to determine the effects of melatonin on body mass and behaviour in irradiated rats.

Methods: Wistar rats were divided into the four experimental groups: I group (control) - rats treated with 0,9\% saline, II group (Mel) - rats treated with melatonin (2 $\mathrm{mg} / \mathrm{kg})$, III group (MW) - rats exposed to MW radiation (4 h/day), IV group (MW+Mel) - rats, which were both exposed to MW radiation and received melatonin premedication $(2 \mathrm{mg} / \mathrm{kg})$.

Results: A significant body mass reduction was noted in animals exposed to MW radiation when compared to controls after 20, 40 and 60 days $(p<0.001)$. Furthermore, body weight was significantly increased $(p<0.05)$ in irradiated rats, which received melatonin pretreatment (MW+Mel) in comparison to irradiated group (MW) after 20 days. Microwave radiation exposed animals showed an anxiety related behaviour (agitation, irritability) after 10 days of exposure. After the radiation source removal, changes in behaviour were less noticeable. Melatonin administration to irradiated rats caused a decrease in the stress induced behaviour.

Conclusion: Microwave radiation causes body mass decrease and anxiety related behaviour in rats, however melatonin causes a reverse of those effects on both body weight and behaviour of irradiated animals (Fig. 2, Ref. 32). Full Text in PDF www.elis.sk.

Key words: melatonin, microwave radiation, mobile phone, body mass, behaviour.
\end{abstract}

Over the last decade, due to the development of new technologies, the number and diversity of appliances (TV sets, mobile phones, PC monitors, etc.) with sources of microwave radiation (MWs) significantly increased. Microwave radiation produced by wireless telecommunications and a number of electrical devices used in household or in healthcare institutions may cause various disorders in human organism. Among the many appliances, due to a rapid expansion of mobile communications and reported symptoms such as headaches and head sensations, memory loss,

${ }^{1}$ Department of Biochemistry, Medical Faculty in Nis, University of Nis,
Nis, Serbia, ${ }^{2}$ Institute of Occupational Health Nis, Medical Faculty in
Nis, University of Nis, Nis, Serbia, ${ }^{3}$ Faculty of Electronic Engineering,
University of Nis, Nis, Serbia, ${ }^{4}$ Institute for Blood Transfusion in Nis,
University of Nis, Nis, Serbia, ${ }^{5}$ Clinic of Dermatology and Venerology,
Clinical Center Niš, Serbia, Serbia, and ${ }^{6}$ Institute for Radiology, Univer-
sity of Nis, Nis, Serbia

Address for correspondence: D. Sokolovic, MD, Department of Biochemistry, Medical Faculty in Nis, University of Nis, Bul. dr Zorana Djindjica 81,18000 Nis, Serbia.

Phone: +381.642136478 , Fax: +381.18238770

Acknowledgement: This work was supported by the Ministry of Science and Technological Development of the Republic of Serbia (Project 43012 and 43011). etc by users, the possible health impairing effects associated with exposure to MWs from mobile handset and base station antenna are particularly of public concern. Nowadays we are faced with growing public concerns about the effects of chronic exposure to MWs $(0.3$ to $300 \mathrm{GHz})$ and theirs' potentially harmful effect on human health (1).

Microwave radiation non-thermal effects cause severe changes in DNA and protein molecules in the brain of experimental animals, micronuclear cell formation in bone marrow, conformational changes in protein molecules and oxidative stress increase in brain and lymphatic tissue $(2,3)$. On the molecular level, these changes cause a development of numerous cellular disorders, such as increase in calcium ions exit in the culture of human neuroblastoma cells (4), reduction in melatonin secretion or disturbances in dopamine-opiate system. Several researches have already shown MW radiation ability to cause DNA damage thereby displaying carcinogenic potential. Lai and Singh (1995) were the first to report that microwave radiation causes dose-dependent interruptions of one or both DNA strands in rat brain cells (5). Oxidative stress is thought to be one of the key mechanisms of cell injury induced by MW radiation on the brain tissue level $(3,6)$. The oxidative stress increase after a MW exposure is probably an important factor for 
apoptosis initiation. Therefore, an increased concentration of $\mathrm{H}_{2} \mathrm{O}_{2}$ and peroxynitrite radicals were found in the majority of apoptotic cells after a MW exposure (3).

It has been proved that MW radiation from mobile phones causes an aggressive behaviour in laboratory animals (6).

Melatonin (N-acetyl-5-methoxytryptamine) is a neurohormone and lipophilic indoleamine derived from tryptophan. In the human body, it is primarily synthesized and released from the pineal gland (epiphysis cerebri) during the night. Melatonin concentration in human blood is significantly higher during the night in comparison to daytime, therefore it is often called the 'hormone of darkness' (7). In this way, melatonin determines the diurnal variations of physiological functions such as sleep, working activity, body temperature, vegetative, digestive and cardiovascular system activity. It has been proved that this hormone slows down or even prevents the process of aging and development of cancer. In small amounts it is also synthesized in the extrapineal tissues such as the retina, ciliary body of the eye, brain tissue, thymus, respiratory epithelium, bone marrow, digestive tract epithelium, ovary, testis, lymphocytes, placenta and skin (8).

Melatonin also shows neuromodulatory functions such as sedative, analgesic, anticonvulsant and hypnotic effect. In vivo experiments in rats have shown that melatonin application increases GABA level in several brain zones (9). Melatonin has a significant anti-oxidative potential (10), and it prevents the oxidative damage of DNA molecules (11).

The aim of this study was to determine changes in body mass (after 20, 40 and 60 days of microwave radiation exposure) and changes in behaviour of experimental animals after a chronic exposure to MW from a mobile phone as well as the effect of melatonin application on body mass and rat behaviour after being exposed to microwaves.

\section{Methods}

\section{Animal model}

Animals used for the procedure were treated in strict accordance with the NIH Guide for Care and Use of Laboratory Animals (1985).

Experiments were performed on 40 adult male Wistar Albino rats, bred in the Vivarium of the Biomedical Research Institute, at Medical faculty, Nis, under conventional laboratory conditions. The control and experimental group animals were collectively housed in plastic cages $30 \times 40 \times 40 \mathrm{~cm}(\mathrm{WxLxH})$ and had ad libitum access to standard laboratory food and water. The housing room was maintained at $24{ }^{\circ} \mathrm{C}$ with $42 \pm 5 \%$ relative humidity and had 12-12-h light-dark cycle (exposure to light from 06:00 AM to $06: 00 \mathrm{PM}$ ).

All experiments on animals had been approved by the Animal Ethics Board of the Medical Faculty in Nis and were performed according to these guidelines.

\section{Microwave radiation exposure}

Animals were exposed to MWs, $4 \mathrm{~h}$ /day during the light period. MWs were produced by a mobile test phone (model NOKIA

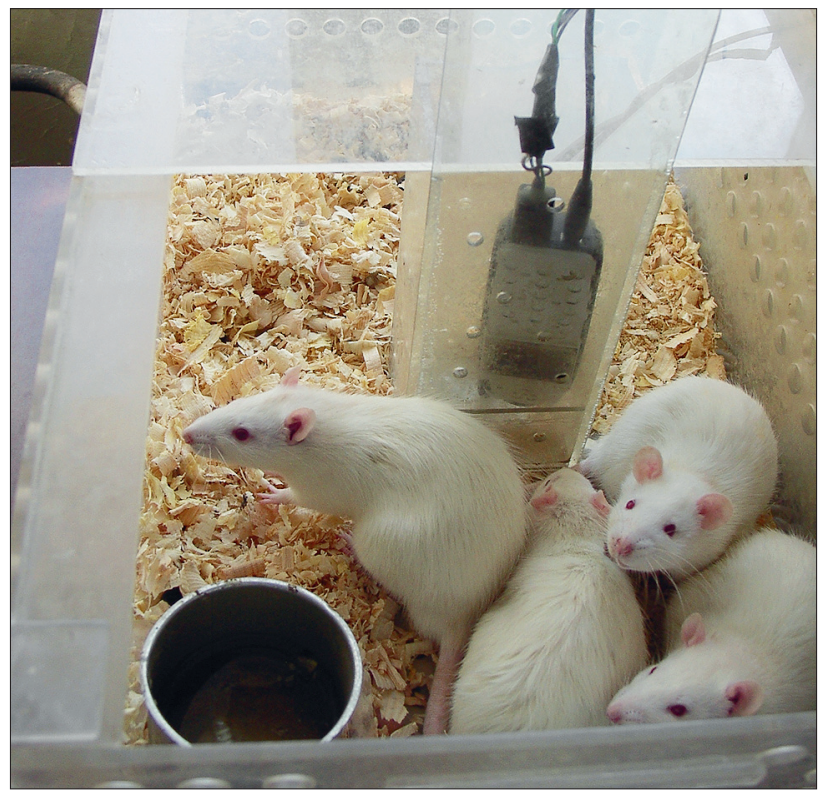

Fig. 1. Experimental animals were placed in cages made of plastic with mobile test phone in the centre.

3110; Nokia Mobile Phones Ltd.) connected to a Communication Test Set PCDK with PC and appropriate software module. The mobile phone was placed in a special protective compartment made of plastic with the antenna positioned downwards (Fig.1). During MW exposure, eight rats were able to move freely in a pure (i.e. lacking any metallic fittings) plastic cage.

An electromagnetic continuous wave near-field signal for GSM (Global System for Mobile communication) at $900 \mathrm{MHz}$ mobile phone system was used for the purpose of this experiment. Electromagnetic field parameters in cage were measured several times during the experimental exposure. Electrical field was estimated by EM field meter AARONIA SPECTRAN HF6080 with E $=9.88$ $\mathrm{V} / \mathrm{m}$ to $18.356 \mathrm{~V} / \mathrm{m}$ and magnetic field $\mathrm{B}=4.68 \mu \mathrm{T}$ to $8.69 \mu \mathrm{T}$. The whole-body specific energy absorption (SAR) rate was estimated to $0.043-0.135 \mathrm{~W} / \mathrm{kg}$ using data for a rotating ellipsoidal rat model.

\section{Experimental design}

Animals were divided into the 4 experimental groups with 10 animals in each group: I group (control) - rats treated with $0.9 \%$ saline, II group (Mel) - rats treated with melatonin $(2 \mathrm{mg}$ $\mathrm{kg}^{-1}$ body weight i.p.), III group (MWs) - MW exposed rats, IV group $(M W s+M e l)-M W$ exposed rats treated with melatonin (2 $\mathrm{mg} \mathrm{kg}^{-1}$ body weight i.p.)

Animals in all experimental groups (groups III and IV) were exposed to microwave radiation for 4 hours every day and then moved to rooms with no sources of electromagnetic field. During exposition, the cages with animals were placed on absorbing material made of rubber with wooden isolation surface, with no electric conductors or metal objects nearby. Chronic exposure to microwave radiation lasted 60 days.

Melatonin was applied intraperitoneally every morning at $08.00 \mathrm{AM}$, at a single dose of $2 \mathrm{mg} / \mathrm{kg}$ body weight $(3,12)$. In 
control rats, isotonic saline solution (equal to the volume of melatonin) was given intraperitoneally everyday during the follow up. Melatonin used for the purpose of this experiment, was of pro analysi purity (ICN Galenika, Belgrade).

\section{Body mass measuring and animal behaviour monitoring}

Body mass (in grams) was measured after 20, 40 and 60 days since the beginning of the experiment.

Rat behaviour was monitored for 20 minutes ( 5 minutes each group), at the very end of the MW exposure period and then 20 minutes after the removal of the electromagnetic field source, every day. The floor surface within a plastic cage, where rats were kept, was approximately divided in 12 quadrants $(10 \times 10 \mathrm{~cm}$ each). Locomotor activity and stress and/or anxiety related behaviour such as moving away from the source of radiation, rearing and grooming were assessed. The number of squares the rat crossed was used as a measure of locomotor activity. Assessment method was based on the Open field test ${ }^{(13)}$, but we tended to minimize the stress caused by moving animals to open field.

\section{Statistical analysis}

Results were presented as means \pm SD. Data were analyzed using the Student's t-test, preformed by means of commercially available statistics software package (SPSS ${ }^{\circledR}$ for Windows, v. 9.0, Chicago, USA). The statistical significance was set to $\mathrm{p}<0.05$.

\section{Results}

Significant body mass reduction was noted in animals exposed to MW radiation, when compared to controls after 20 days (161.7 \pm 9.3 vs $190.0 \pm 4.5 \mathrm{~g}, \mathrm{p}<0.001), 40$ days $(185.8 \pm 22.7$ vs $276.7 \pm 15.4 \mathrm{~g}, \mathrm{p}<0.001)$ and 60 days $(257.7 \pm 18.9 \mathrm{vs} 310.0 \pm 20.7 \mathrm{~g}$, $\mathrm{p}<0.001$ ) of experiment, as shown in Figure 2 .

It has also been observed that the intraperitoneal application of melatonin caused significant body mass increase in rats, when compared to the control group $(257.5 \pm 9.3 \mathrm{vs} 190.0 \pm 4.5 \mathrm{~g}$, $\mathrm{p}<0.001$ ) after 20 days.

Furthermore, body weight was significantly increased in irradiated rats, which received melatonin pretreatment $(\mathrm{MW}+\mathrm{Mel})$ in comparison to irradiated rats (MW) after 20 days (180.0 \pm 7.1 vs $161.7 \pm 9.3 \mathrm{~g}, \mathrm{p}<0.05)$. Similar tendency was observed after 40 and 60 days of experiment but these changes were not statistically significant (Fig. 2).

Microwave radiation exposed animals showed changes in behaviour (agitation, anxiety, irritability) after 10 days of exposure. Further increase in MW exposure duration caused more intense changes in animal behaviour. The observed changes were the most intense after 60 days of MW exposure. Melatonin application to irradiated animals reduced the stress and anxiety related behaviour.

Rats exposed to MWs tended to move away from the source of radiation, which was placed in the centre of the plastic cage what was manifested as grouping in outer quadrants. Locomotor activity of MW exposed rats (group III and IV) was slightly reduced when compared to unexposed animals (group I and II). On

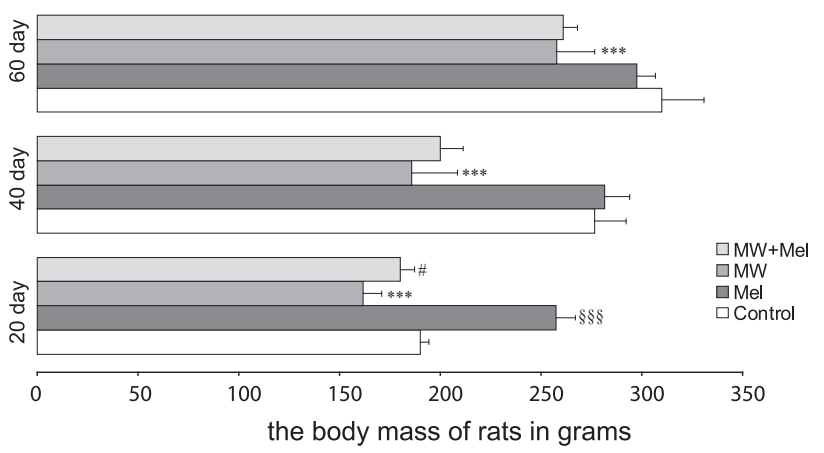

Fig. 2. Body mass (in grams) of rats (control group; Mel-melatonin treated group; MW - MW exposed group; MW+Mel - MW exposed group with melatonin pretreatment). ${ }^{* * *} \mathbf{p}<\mathbf{0 . 0 0 1}$ vs Control; ${ }^{*} \mathbf{p}<\mathbf{0 . 0 5}$ vs $\mathrm{MW} ;{ }^{\S \S} \mathbf{p}<\mathbf{0 . 0 0 1}$ vs Control, MW and MW+Mel.

the other hand, we observed no significant changes among MW exposed animals who either received or did not receive melatonin pretreatment.

MW exposed rats showed a significantly less exploratory behaviour assessed trough reduced rearing when compared to unexposed animals and exposed animals who received melatonin pretreatment. MW exposed and not treated with melatonin rats spent more time grooming when compared to other groups. After the electromagnetic field source removal, the changes in behaviour were less noticeable.

\section{Discussion}

Animals exposed to microwave radiation showed changes in behaviour such as decreased locomotor activity and a higher degree of anxiety. Animals showed an obvious tendency to group as far as possible from the source of radiation. At the same time, the exploratory behaviour such as rearing was reduced, which can be interpreted as a sign of stress. An increased grooming as well may indicate a self-soothing behaviour in stressed animals. Melatonin had no effect on the locomotor activity, jet it reduced stress and anxiety associated behaviour in rats. However, we emphasize the fact that the behaviour parameters can be altered in both ways (reduced or increased) in animals under stress. At the same time, the irradiated animals showed less body mass gain, which was corrected by melatonin administration, but only during 20 days of experiment, whereas the long-term application of melatonin had no significant effect on body mass gain. Sandrey et al (2002) reported that electromagnetic field shows predominantly a short term effect on body mass in rats (14). They reported an increased mass loss and increased duration of mass-loss in young rats, but in the initial period of 21 day long experiment. Therefore, melatonin protective effects are the most obvious in the initial period of stress exposure due to an increase mass loss in the same period.

Behaviour disorders detected in animals during MW exposure can be partially ascribed to the disorders in the electroencephalographic (EEG) activity. On the other hand, these disorders, such as increase in spectral strength and irregularities in EEG findings, are 
consequences of an increased amount of nitric oxide (NO) under the effect of microwave radiation (15). The signal transduction begins after calcium is being released by neurons under the effect of MW, whereby glutamine receptor is activated and the activity of nitric oxide synthase (NOS) is increased, along with the synthesis of NO and citrulline. Nitric oxide is a gaseous and easily diffusible molecule therefore it passes through cellular membranes easily and stimulates conversion of GTP to cGMP leading to consequential biochemical disorders. At the same time, it is being converted into a high toxic peroxynitrite (ONOO-) due to the oxidative stress caused by MWs exposure.

It has been proved that some brain structures (globus pallidus, substantia nigra, hypothalamus) are particularly sensitive to the reactive oxygen species (ROS). It has also been confirmed that brain tissue contains a small amount of antioxidant protection enzymes (16). Adisturbance of these functions in the brain causes disorders in the sphere of an affective behaviour as well as disorders of neurovegetative functions, which can explain the stated changes in behaviour and dietary habits in animals exposed to MW radiation. This points to a possible mechanism of an increased sensitivity to electromagnetic fields (EMFs) in some structures of the central nervous system (CNS), primarily hypothalamus and hypophysis (17).

The changes in behaviour of tested animals could be partially explained by numerous biological effects of MW radiation in the frequency range from $2.45 \mathrm{GHz}$ to $27 \mathrm{MHz}$. It is commonly stated that MW radiation cause disorders in the ion channel functions, such as reduced production of canalicular membrane proteins, reduced ion channel production and an increased rate of sudden opening (18). Literature data reported an increased calcium ion exit from neurons in rats exposed to microwave radiation with the frequency of $2.45 \mathrm{GHz}$ (19). Microwave radiation also causes conformational changes in proteins, $\beta$-lactoglobulins (20) and increased permeability of the blood-brain barrier (21).

Literature data show that MW radiation may cause abnormal brain functioning. Study conducted by Lai and Sigh (1999) lead to the conclusion that spatial orientation can be disturbed when SAR values are below $1 \mathrm{~W} / \mathrm{kg}(22)$. It has been recently reported that GSM $900 \mathrm{MHz}$ radiation (SAR 0.41-0.98 W/kg) causes deficits in consolidation and/or retrieval of the learned spatial information (23). On the other hand, Jadidi et al (2009) reported the opposite findings on this matter, therefore further investigation regarding this problem is needed (1). Not only spatial memory but also non-spatial memory may be affected by EMF exposure. Ntzouni et al (2010) have reported severe disruptions in the consolidation phase of recognition memory processes while performing Object Recognition Task test in mice (24). Therefore they believe that the primary EMF target may be the information transfer pathway connecting the entorhinal-parahippocampal regions, which participate in the ORT memory task. Narayanan et al (2010) reported that EMF exposure significantly altered the passive avoidance behaviour and hippocampal morphology in rats (25). Hippocampal region is taught to be crucial in the learning process and spatial memory formation. Long-term potentiation is an attractive hypothesis, which might explain cellular mechanisms of relational learning. However, Jadidi et al (2007) found no evidence to sup- port the theory that the long-term potentiation is affected by the whole-body exposure to low-power density of $950 \mathrm{MHz}$ field of GSM mobile phone system (26). D'Andrea (1999) had shown in mice that cognitive tasks demonstrated an extreme sensitivity to MW exposure and that disturbances in cognitive functions are expressed more than disorders in some simple tasks that are learned in advance (27). It seams that there is a lot of controversy surrounding this field of research nowadays. Therefore, there is a strong need for further research in this area, having in mind that number of mobile phone users is rapidly increasing all over the world.

Melatonin effect on EMF induced neurobehavioral disorders has been confirmed by the numerous preclinical studies (28), which have shown that melatonin acts as antidepressant in rodents. This explains an increase in body mass of rats in the melatonin treated group. Szemerszky et al (2009) reported a hormonal stress reaction and a depression like behaviour after the long term exposure to microwaves in rats (29). Latest researches show that this effect is mediated by N-methyl D-aspartate (NMDA) receptor complex, which takes a part in the pathogenesis of depression and anxiety. The evidence in favour of this claim can be found in the fact that NMDA receptor antagonists have antidepressant effects in both preclinical and clinical tests (30). There is an evidence that L-arginine/NO is also included in the depression pathogenesis. The inhibition of cerebral NOS by melatonin is another possible antidepressant mechanism (28), which has been confirmed in this research.

The pineal gland activity and melatonin secretion are not only influenced by the external light sources but they also depend on the EMF. Brendal et al (2000) showed changes in the pineal gland activity exposed to constant electromagnetic field of similar intensity as Earth's geomagnetic field $(35 \mu \mathrm{T})(31)$. Based on this results, they assumed that the pineal gland serves as a geomagnetic compass in human body and that it is important for a spatial orientation of an individual (31). This assumption was later confirmed by researches, which showed that changes in a geomagnetic field caused a reduction of the melatonin secretion (30). The experimental study conducted by Semm et al (1980) had shown that a weak magnetic field causes a significant reduction of melatonin probably by reducing the $\mathrm{N}$-acetyltransferase activity (32).

In conclusion, microwave radiation causes body mass decrease and anxiety related behaviour in rats, however melatonin causes a reverse of those effects on both body weight and behaviour of irradiated animals.

\section{References}

1. Jadidi M, Firoozabadi SM, Rashidy-Pour A, Bolouri B, Fathollahi Y, Sajadi AA. Does whole body exposure to GSM-950 MHz electromagnetic fields affect acquisition and consolidation of spatial information in rats? Iran J Radiat Res 2009; 7 (1): 57-62.

2. Demsia G, Vlastos D, Matthopoulos DP. Effect of 910-MHz electromagnetic field on rat bone marrow. Scientific World Journal 2004; 4 (2): 48-54.

3. Sokolovic D, Djindjic B, Nikolic J, Bjelakovic G, Pavlovic D, Kocic G, Krstic D, Cvetkovic T, Pavlovic V. Melatonin reduces oxidative stress induced by chronic exposure of microwave radiation from mobile phones in rat brain. J Radiat Res 2008; 49 (6): 579-586. 
4. Dutta SK, Subramoniam A, Ghosh B, Parshad R. Microwave radiation-induced calcium ion efflux from human neuroblastoma cells in culture. Bioelectromagnetics 1984; 5 (1): 71-78.

5. Lai H, Singh NP. Acute low-intensity microwave exposure increases DNA single-strand breaks in rat brain cells Bioelectromagnetics 1995; 16 (3): 207-210.

6. Tian F, Nakahara T, Yoshida M, Honda N, Hirose H, Miyakoshi J. Exposure to power frequency magnetic fields suppresses X-ray-induced apoptosis transiently in Ku80-deficient xrs5 cells. Biochem Biophys Res Commun 2002; 292 (2): 355-361.

7. Reiter RJ. Pineal melatonin: cell biology of its synthesis and of its physiological interactions. Endocr Rev 1991; 12: 151-180.

8. Sahelian R. Melatonin. Arch Fam Med 1996; 5 (4): 196.

9. Xu F, Li JC, Ma KC, Wang M. Effects of melatonin on hypothalamic gamma-aminobutyric acid, aspartic acid, glutamic acid, beta-endorphin and serotonin levels in male mice. Biol Signals 1995; 4 (4): 225-231.

10. Poeggeler B, Reiter RJ, Tan DX, Chen LD, Manchester LC. Melatonin, hydroxyl radical-mediated oxidative damage, and aging: a hypothesis. J Pineal Res 1993; 14 (4): 151-168.

11. Jou MJ, Peng TI, Yu PZ, Jou SB, Reiter RJ, Chen JY, Wu HY, Chen CC, Hsu LF. Melatonin protects against common deletion of mitochondrial DNA-augmented mitochondrial oxidative stress and apoptosis. J Pineal Res 2007; 43 (4): 389-403.

12. Drago F, Frisina M, Grech M, Nicolosi A, Micale V, Nicosia A, Medico M, Foti F. Dual effects of melatonin on barbiturate-induced narcosis in rats. Neurosci Lett 2001; 300 (3): 176-178.

13. Prut L, Belzung C. The open field as a paradigm to measure the effects of drugs on anxiety-like behaviors: a review. Eur J Pharmacol 2003; 463 (1-3): 3-33.

14. Sandrey MA, Vesper DN, Johnson MT, Nindl G, Swez JA, Chamberlain J, Balcavage WX. Effect of Short Duration Electromagnetic Field Exposures on Rat Mass. Bioelectromagnetics 2002; 23 (1): 2-6

15. Bawin SM, Satmary WM, Jones RA, Adey WR, Zimmerman G. Extremely low frequency magnetic fields disrupt rhythmic slow activity in rat hippocampal slices. Bioelectromagnetics 1996, 17 (5): 388-395.

16. Abdel-Rassoul G, El-Fateh OA, Salem MA, Michael A, Farahat F, El-Batanouny M, Salem E. Neurobehavioral effects among inhabitants around mobile phone base stations. Neurotoxicology 2007; 28 (2): 434-440.

17. Burch JB, Reif JS, Yost MG. Geomagnetic activity and human melatonin metabolite excretion. Neurosci Lett 2008; 438 (1): 76-79.

18. Repacholi MH. Low level exposure to radiofrequency electromagnetic fields: health effects and research needs. Bioelectromagnetics 1998; 19 (1): 1-19.
19. Paulraj R, Behari J. The effect of low level continuous $2.45 \mathrm{GHz}$ wave on brain enzymes of developing rat brain. Electromag Biol Med 2002; 21 (3): 231-241.

20. Bohr H, Bohr J. Microwave enhanced kinetics observed in ORD studies of protein, Bioelectromagnetics 2000; 21 (1): 68-72.

21. Neubauer C, Phelan AM, Kues H, Lange DG. Microwave irradiation of rats at $2.45 \mathrm{GHz}$ activates pinocytotic-like uptake of tracer by capillary endothelial cells of cerebral cortex. Bioelectromagnetics 1990; 11 (4): 261-268.

22. Lai H, Singh NP. Single-and double-strand DNA breaks in rat brain cells after acute exposure to radiofrequency electromagnetic radiation. Int J Radiat Biol 1999; 69 (4): 513-521.

23. Fragopoulou AF, Miltiadous P, Stamatakis A, Stylianopoulou F, Koussoulakos SL, Margaritis LH. Whole body exposure with GSM $900 \mathrm{MHz}$ affects spatial memory in mice. Pathophysiology 2010; 17 (3): 179-187.

24. Ntzouni MP, Stamatakis A, Stylianopoulou F, Margaritis LH. Shortterm memory in mice is affected by mobile phone radiation. Pathophysiology 2011; 18 (3): 193-199.

25. Narayanan SN, Kumar RS, Potu BK, Nayak S, Bhat PG, Mailankot M. Effect of radio-frequency electromagnetic radiations (RF-EMR) on passive avoidance behaviour and hippocampal morphology in Wistar rats. Ups J Med Sci 2010; 115 (2): 91-96.

26. Jadidi M, Firoozabadi SM, Rashidy-Pour A, Bolouri B, Fathollahi Y. Low-power density of $950 \mathrm{MHz}$ radiation does not affect long-term potentiation in rat dentate gyrus. Iran J Radiat Res 2007; 5 (3): 119-124.

27. D’Andrea JA. Behavioural evaluation of microwave irradiation. Bioelectromagnetics 1999; 20 (4): 64-74.

28. Mantovani M, Pertile R, Calixto J, Santos A, Rodrigues ALS. Melatonin exerts an antidepressant-like effect in the tail suspension test in mice: evidence for involvement of N-methyl-D-aspartate receptors and the Larginine-nitric oxide pathway. Neuroscience Letters 2003; 343 (1): 1-4.

29. Szemerszkya R, Zelenab D, Barnab I, Bárdosc G. Stress-related endocrinological and psychopathological effects of short- and long-term $50 \mathrm{~Hz}$ electromagnetic field exposure in rats. Brain Research Bulletin 2010; 81 (1): 92-99.

30. Skolnick P. Antidepressants for the new millennium. Eur J Pharmacol 1999; 375 (1-3): 31-40.

31. Brendel H, Niehaus M, Lerchl A. Direct suppressive effects of weak magnetic fields $(50 \mathrm{~Hz}$ and $162 / 3 \mathrm{~Hz})$ on melatonin synthesis in the pineal gland of Djungarian hamsters (Phodopus sungorus). J Pineal Res 2000; 29 (4): 228-233.

32. Semm P, Schneider T, Vollrath L. Effects of an earth-strength magnetic field on electrical activity of pineal cells. Nature 1980; 288 (5791): 607-608. 